\title{
Genetic Causes of Clopidogrel Nonresponsiveness: Which Ones Really Count?
}

\author{
Kathryn M. Momary, Pharm.D., Michael P. Dorsch, Pharm.D., M.S., and Eric R. Bates, M.D.
}

Clopidogrel decreases the morbidity and mortality associated with several cardiovascular diseases. However, clopidogrel is a prodrug that needs to be metabolized to the active thiol metabolite by the cytochrome P450 (CYP) system. This activation is a source of significant interindividual variability in clopidogrel responsiveness. Drug interactions with and genetic variation in CYP3A4, CYP3A5, and CYP2C19 enzymes have been implicated in decreasing active metabolite production. In addition, polymorphisms in the genes encoding P-glycoprotein (an efflux transporter) and purinergic receptor $\mathrm{P}_{2} \mathrm{Y}_{12}$ (the active site for clopidogrel) have been studied for their role in clopidogrel responsiveness. Several large studies have recently assessed the role of genetic variation in clopidogrel responsiveness as characterized by clinical outcomes. In this review, we summarize the genetic causes of clopidogrel nonresponsiveness, with a focus on larger outcomes-based studies. A MEDLINE search of the English-language literature (1990-2008) was conducted to identify studies that examined these relationships; additional citations were obtained from the articles retrieved from the literature search. Polymorphisms in CYP2C19 and, to a lesser extent, the adenosine $5^{\prime}$-triphosphate-binding cassette gene, $A B C B 1$, contribute to variability in clopidogrel responsiveness. Specifically, patients possessing at least one variant CYP2C19 allele $($ CYP2C19*2, *3) have impaired clopidogrel responsiveness due to decreased formation of the active metabolite. In addition, one study found that considering $A B C B 1$ genotype in addition to CYP2C19 allowed better prediction of clopidogrel nonresponsiveness. However, routine genotyping for CYP2C19 or ABCB1 polymorphisms in order to predict clopidogrel responsiveness cannot be recommended at this time because of logistic and cost considerations.

Key Words: clopidogrel, pharmacogenetics, cytochrome P450, CYP2C19, $A B C B 1$.

(Pharmacotherapy 2010;30(3):265-274)

\section{OUTLINE}

Genetic Causes of Clopidogrel Nonresponsiveness CYP2C19

CYP3A4/5

$A B C B 1$

P2RY12

Clinical Relevance

Patients at Risk for Decreased Response to Clopidogrel

Routine Genotyping for CYP2C19 Variant Alleles to Predict Clopidogrel Responsiveness: Logistic and Cost Considerations

Conclusion
Clopidogrel decreases the morbidity and mortality associated with several cardiovascular diseases. The Clopidogrel versus Aspirin in Patients at Risk for Ischemic Events (CAPRIE) trial demonstrated that clopidogrel improves outcomes in patients with a history of recent ischemic stroke, myocardial infarction, or symptomatic peripheral artery disease. ${ }^{1}$ Subsequently, the Clopidogrel in Unstable Angina to Prevent Recurrent Events (CURE) trial demonstrated that the addition of clopidogrel improved outcomes in patients with non-STsegment elevation acute coronary syndromes 
(ACS). ${ }^{2}$ The Clopidogrel for Reduction of Events During Observation (CREDO) trial found similar results in patients undergoing percutaneous coronary intervention (PCI). ${ }^{3}$ These trials resulted in the American College of CardiologyAmerican Heart Association guideline recommendations that clopidogrel be used in combination with aspirin for ACS and PCI., 5 Comprehensive recommendations for clopidogrel use in this setting have been published previously. ${ }^{6}$

Despite the well-documented benefits of clopidogrel, there is significant interindividual variability in clopidogrel responsiveness. This variability leads to some patients having decreased inhibition of platelet aggregation with clopidogrel, or nonresponsiveness, and this has been associated with increased risk for cardiovascular events. ${ }^{7,8}$ Some studies have used ex vivo platelet aggregation testing whereas others have used increased rates of adverse cardiovascular events to characterize variability in response to clopidogrel.

The primary source of the variability in clopidogrel responsiveness lies in the pharmacokinetics of clopidogrel. Clopidogrel is a prodrug that needs to be metabolized to the active thiol metabolite by the cytochrome P450 (CYP) system (Figure 1). This metabolite then irreversibly inhibits the purinergic receptor $\mathrm{P} 2 \mathrm{Y}_{12 .}{ }^{9}$ In recent years, significant interindividual variability in clopidogrel responsiveness has been noted. Drug interactions with and genetic variation in CYP3A4, CYP3A5, and CYP2C19 enzymes have been implicated in decreased active metabolite production. This has resulted in a recent change in the clopidogrel prescribing information, which now includes information on CYP2C19 genotyping and concomitant use of CYP2C19 inhibitors. ${ }^{10}$

A number of ongoing studies are using different dosing strategies to overcome some of

From the Department of Pharmacy Practice, Mercer University College of Pharmacy and Health Sciences, Atlanta, Georgia (Dr. Momary); and the Department of Pharmacy Services (Dr. Dorsch) and the Division of Cardiovascular Medicine, Department of Internal Medicine (Dr. Bates), University of Michigan Health System, and the Department of Clinical, Social, and Administrative Sciences, University of Michigan College of Pharmacy (Dr. Dorsch), Ann Arbor, Michigan.

For reprints, visit http://www.atypon-link.com/PPI/loi/phco. For questions or comments, contact Kathryn M. Momary, Pharm.D., BCPS, Mercer University College of Pharmacy and Health Sciences, Department of Pharmacy Practice, 3001 Mercer University Drive, Atlanta, GA 30341-4155; email: momary_km@mercer.edu. the interindividual variability associated with clopidogrel CYP drug interactions and genetic variability. In the meantime, it is important that clinicians understand the mechanisms of clopidogrel interindividual variability. In this review, we summarize the genetic causes of clopidogrel nonresponsiveness, with a focus on larger clinical outcome-based studies. A MEDLINE search of the English-language literature (1990-2008) was conducted to identify studies that examined these relationships; additional citations were obtained from the articles retrieved from the literature search.

\section{Genetic Causes of Clopidogrel Nonresponsiveness}

\section{CYP2C19}

Several studies have assessed the role of the CYP2C19 genotype in clopidogrel responsiveness. The CYP2C19*2 allele, along with the *3,*4, and *5 alleles, have been associated with decreased metabolic activity and have thus been termed loss-of-function alleles (Table 1). The CYP2C19*2 allele is the most frequently occurring variant allele in Caucasian, African-American, and Asian populations. However, the allele frequency differs by racial group (Figure 2). ${ }^{11,12}$ The CYP2C19*2 allele frequency in Asian populations $(\sim 30 \%)$ is significantly higher than that seen in Caucasians ( $13 \%)$ and African-Americans $(\sim 18 \%)$. The CYP2C19*3 allele occurs more frequently in Asian populations ( 10\%) compared with other racial groups $(<1 \%)$.

Several studies have demonstrated that CYP2C19 genotype affects the pharmacokinetics and pharmacodynamics of clopidogrel. ${ }^{13-15}$ Findings in smaller pharmacokinetic and pharmacodynamic studies have now been confirmed in large cohort studies (Table 2). ${ }^{15-21}$

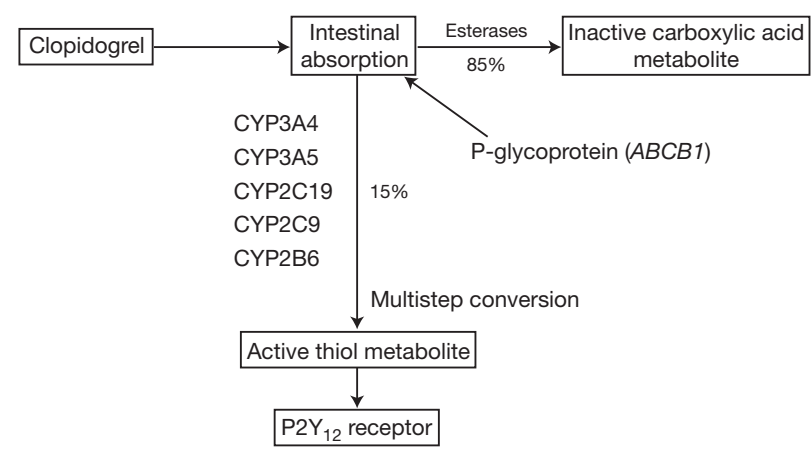

Figure 1. Metabolism of clopidogrel. CYP = cytochrome P450. 
Table 1. Genes That Encode the Proteins Involved in Clopidogrel Pharmacokinetics and Pharmacodynamics

\begin{tabular}{llll}
\hline Gene & Protein Encoded & SNPs & $\begin{array}{c}\text { Relative Change in } \\
\text { Protein Function }\end{array}$ \\
\hline CYP2C19 & CYP2C19 & $* 1$ & Wild-type \\
& $* 2$ & Decreased activity \\
& $* 3$ & Decreased activity \\
& $* 4$ & Decreased activity \\
& & $* 5$ & Decreased activity \\
CYP3A4 & CYP3A4 & $* 1 B$ & Wild-type \\
& & $23171 \mathrm{~T}>\mathrm{C}$ & Unknown activity \\
& & IVS7+258A $>\mathrm{G}$ & Unknown activity \\
& & IVS7+894C $>\mathrm{T}$ & Unknown activity \\
CYP3A5 & IVS10+12G $>$ A & Unknown activity \\
ABCB1 & & $* 1$ & Wild-type \\
& & $* 3$ & Decreased activity \\
P2RY12 & & $3435 \mathrm{C}>\mathrm{T}$ & Unknown activity \\
& & $2677 \mathrm{G}>\mathrm{T}$ & Unknown activity \\
PNP & & P2 & Unknown activity \\
& & $744 \mathrm{~T}>\mathrm{C}$ & Unknown activity \\
\hline
\end{tabular}

SNP = single nucleotide polymorphism; CYP = cytochrome P450; IVS = intervening sequence; $\mathrm{ABC}=$ adenosine $5^{\prime}$-triphosphate-binding cassette; $\mathrm{P}^{2} \mathrm{Y}_{12}=$ purinergic receptor .

The association between CYP2C19 genotype and a composite end point of cardiovascular death, nonfatal myocardial infarction, and urgent revascularization was studied in a prospective cohort study that consisted of 259 young patients who survived a first myocardial infarction, received clopidogrel $75 \mathrm{mg} / \mathrm{day}$, and were enrolled in a multicenter registry 3 months after the acute coronary event. ${ }^{18}$ Patients possessing the CYP2C19*2 allele (28\%), compared with CYP2C19 wild-type homozygotes (72\%), had a significant increase in the risk for the primary end

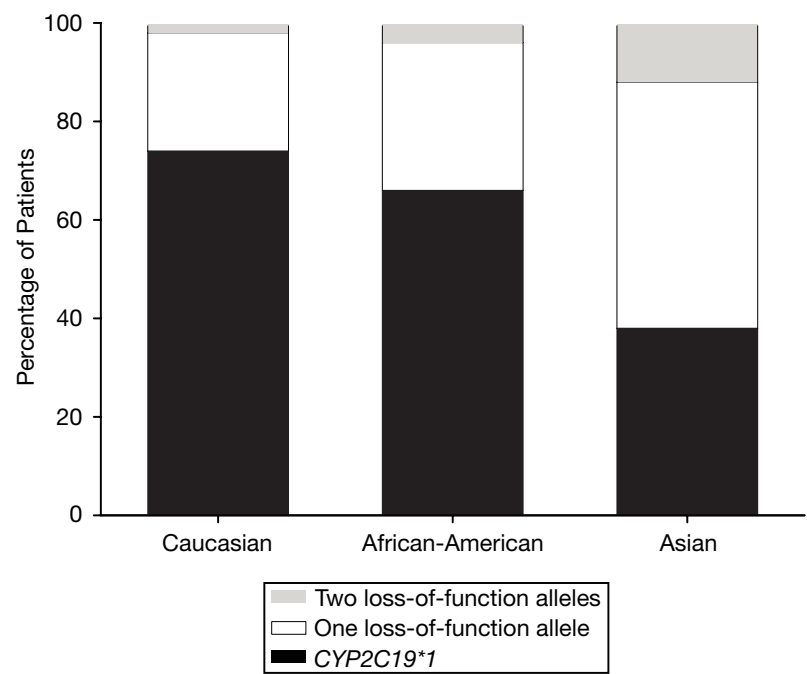

Figure 2. Distribution of CYP2C19 genotype frequencies by race. ${ }^{11,12}$ point at 1 year. This increased risk was demonstrated early after clopidogrel initiation and persisted throughout the study period. An increased risk for stent thrombosis was also found in CYP2C19*2 allele carriers. A multivariable analysis suggested that CYP2C19 genotype was the only significant predictor of the primary outcome in this population. Of note, this study population was fairly heterogeneous, and patients were not randomized to a specific treatment strategy. However, these results suggest that possession of at least one variant CYP 2 C $19 * 2$ allele is associated with increased risk.

Another study assessed a cohort of 2208 patients from the prospective, observational French Registry of Acute ST-Elevation and Non-ST-Elevation Myocardial Infarction (FASTMI) registry who had received clopidogrel and contributed a DNA sample. ${ }^{19}$ All patients had documented myocardial infarction, were admitted to the intensive care unit, and underwent genotyping for polymorphisms in the following genes: CYP2C19; CYP3A5; the adenosine $5^{\prime}$-triphosphate-binding cassette gene, $A B C B 1$; the $\mathrm{P}_{2} \mathrm{Y}_{12}$ gene, $\mathrm{P} 2 \mathrm{RY} 12$; and the integrin $\beta_{3}$ gene, ITGB3 (IIb-IIIa receptor). Unlike the above-mentioned study, ${ }^{18}$ in this study genotyping for CYP2C19*2, *3,*4, and *5 alleles was performed. This study also used a composite primary outcome, which included death from any cause, nonfatal stroke, or myocardial infarction. Many demographic and clinical characteristics differed between those patients 
Table 2. Clopidogrel-CYP2C19 Pharmacogenomic Studies That Evaluated Clinical Outcomes

\begin{tabular}{lll}
\hline Population & Gene and Alleles & Outcome Variable Studied \\
\hline $\begin{array}{l}\text { Patients undergoing elective coronary } \\
\text { stent placement and received a routine } \\
\text { clopidogrel 600-mg loading dose } \\
(\mathrm{n}=797)^{16}\end{array}$ & CYP2C19*1, ${ }^{*}$ & $\begin{array}{l}\text { On-treatment platelet reactivity and association of } \\
\text { platelet reactivity with risk of death or myocardial } \\
\text { infarction }\end{array}$ \\
& &
\end{tabular}

Patients with STEMI undergoing fibrinolysis with scheduled angiography and a clopidogrel 300-mg loading dose $(\mathrm{n}=465)^{17}$

Patients aged 18-45 yrs who survived their first myocardial infarction and received clopidogrel $75 \mathrm{mg} /$ day for at least 1 mo with an average duration of clopidogrel exposure of 1.07 yrs $(\mathrm{n}=259)^{18}$

Patients admitted to the intensive care unit after a myocardial infarction $(\mathrm{n}=2208)^{19}$

Patients undergoing primary PCI and received a routine clopidogrel 600-mg loading dose $(n=2485)^{20}$

Patients with ACS and planned PCI from TRITON-TIMI 38, and received a clopidogrel 300-mg loading dose $(\mathrm{n}=1477)^{15}$

Patients receiving drug-eluting stents and received a routine clopidogrel 600-mg loading dose $(\mathrm{n}=772)^{21}$
CYP $2 C 19 * 1, * 2$,

CYP1A2, CYP2C9,

CYP3A4, CYP3A5

CYP2C19*1,*2

CYP $2 C 19 * 1, * 2, * 3$

*4, *5, CYP3A5,

ABCB1, P2RY12,

ITGB3

CYP $2 C 19 * 1, * 2$

CYP2C $19 * 1, * 2, * 3$,

$* 4, * 5, * 6, * 7, * 8, * 9$,

$* 10, * 11, * 12, * 13$,

$* 14, * 17$, CYP 2 C 9 , CYP2B6, CYP3A5,

CYP3A4, CYP1A2

CYP $2 C 19 * 1,{ }^{*} 2$
Occluded infarct-related artery, death, or recurrent myocardial infarction before angiography

Composite end point of death, nonfatal myocardial infarction, and urgent revascularization

Composite end point of death from any cause, nonfatal stroke, or myocardial infarction during 1 yr of follow-up

Definite stent thrombosis within the first 30 days after PCI

Composite end point of death from cardiovascular causes, myocardial infarction, or stroke $15 \mathrm{mo}$ after randomization

Definite or probable stent thrombosis within 6 mo of PCI

CYP = cytochrome P450; HR = hazard ratio; CI confidence interval; STEMI = ST-segment elevation myocardial infarction; PCI = percutaneous coronary intervention; TRITON-TIMI = Trial to Assess Improvement in Therapeutic Outcomes by Optimizing Platelet Inhibition with Prasugrel-Thrombolysis in Myocardial Infarction; OR = odds ratio.

who had the primary outcome and those who did not. Specifically, those who had the primary outcome (294 patients [13\%]) were older, had more comorbidities, were more likely to be receiving drugs for secondary prevention (including clopidogrel) before their myocardial infarction, were less likely to receive primary PCI or intravenous fibrinolysis during the inclusion event, and were less likely to have received statins, $\beta$-blockers, angiotensin-converting enzyme inhibitors, glycoprotein IIb-IIIa inhibitors, or heparin during the event. No single nucleotide polymorphisms in CYP3A5, P2RY12, or ITGB3 were associated with the primary outcome. Patients possessing two variant CYP2C19 alleles were more likely to experience a primary outcome event compared with noncarriers. Similarly, those possessing two
ABCB1 3435T alleles had an increased risk for a primary outcome event compared with noncarriers. Those patients who possessed two CYP2C19 loss-of-function alleles and at least one $A B C B 1$ variant allele were at the highest risk for a primary outcome event (hazard ratio [HR] 5.31, 95\% confidence interval [CI] 2.13-13.20) compared with patients who were both CYP2C19 and $A B C B 1$ wild-type homozygotes. When the analysis was limited to the 1535 patients who underwent PCI, the association with CYP2C19 genotype persisted, whereas the $A B C B 1$ genotype was no longer significantly associated with risk of the primary outcome. This study was not randomized and is confounded by the fact that patients who had an event were more likely to be receiving clopidogrel at baseline. Thus, a group of clopidogrel nonresponders may have been 
Table 2. (continued)

\begin{tabular}{l}
\hline Results \\
\hline Patients with at least one CYP2C19*2 allele had increased \\
platelet reactivity \\
Increased platelet reactivity was associated with an increased \\
risk of death or myocardial infarction (HR 3.0, 95\% CI \\
$1.4-6.8$ ) \\
CYP2C19 genotype was not associated with risk of death or \\
myocardial infarction \\
No significant interactions between evaluated gentoypes and \\
composite end point $(\mathrm{p}>0.3)$
\end{tabular}

Patients with at least one CYP2C19*2 allele had an increased risk for the composite end point (HR 3.69, 95\% CI 1.69-8.05)

Patients with two CYP2C19*2,*3,*4, or *5 alleles and those with two variant $A B C B 13435 \mathrm{~T}$ alleles had increased risk for the composite outcome (HR 1.98, 95\%CI 1.10-3.58 and HR 1.72, 95\% CI 1.20-2.47, respectively)

Patients with at least one CYP2C19*2 allele had an increased risk for stent thrombosis (HR 3.81, 95\% CI 1.45-10.02)

Patients with at least one CYP2C19 loss-of-function allele had an increased risk for the composite end point (HR 1.53, 95\% CI 1.19-8.0)

Patients with at least one CYP2C19*2 allele had an increased risk for stent thrombosis (OR 2.59, 95\% CI 1.15-5.88)

preselected and overrepresented in this study. This study, in contrast to the previously mentioned study, ${ }^{18}$ does not clearly define the role of heterozygous genotypes for $A B C B 1$ or CYP2C19 in this patient population.

Another large cohort study assessed 2485 consecutive patients undergoing PCI who were uniformly pretreated with a clopidogrel $600-\mathrm{mg}$ loading dose. ${ }^{20}$ In this study, genotyping was performed only for the CYP2C19*1 and *2 alleles. The primary aim of the study was to determine whether CYP2C19 genotype was associated with risk of stent thrombosis in the 30 days after PCI, defined as the occurrence of ACS with either angiographic or pathologic confirmation of thrombosis. Stent thrombosis occurred in $0.7 \%$ (17 patients). The CYP 2 C $19 * 2$ allele carriers (680 patients [27\%]) had a higher risk for stent thrombosis than did wild-type homozygotes ( $1.5 \%$ vs $0.4 \%$ ). On multivariable analysis, CYP2C19 genotype was the only independent predictor of stent thrombosis risk. Although this study was not randomized, all patients were treated uniformly with PCI and standard clopidogrel doses. It does suggest that patients undergoing PCI who possess at least one CYP2C19*2 allele are at increased risk for stent thrombosis.

The role of the CYP2C19 genotype in clopidogrel responsiveness was also assessed in the Low Responsiveness to Clopidogrel and Sirolimus- or Paclitaxel-Eluting Stent Thrombosis (RECLOSE) trial. ${ }^{21}$ The study included 772 consecutive patients receiving drug-eluting stents and dual antiplatelet therapy with aspirin and clopidogrel. All subjects received a clopidogrel 600-mg loading dose and a 75-mg/day maintenance dose. Genotyping for CYP2C19*1 and *2 alleles was performed. The primary end point was definite or probable stent thrombosis during 6 months of follow-up. Patients carrying at least one variant CYP2C19*2 allele were more likely to experience stent thrombosis. In addition, CYP2C19 genotype was found to be an independent predictor of stent thrombosis risk after inclusion of several clinical risk factors in a multivariable logistic regression model. Similar to the abovementioned study, ${ }^{20}$ this would suggest that patients with at least one CYP2C19 variant allele are at increased risk for stent thrombosis.

All of the studies assessing CYP2C19 genotype and clopidogrel responsiveness discussed thus far have been cohort studies. One group of investigators addressed this question by using data from a randomized controlled trial. ${ }^{15}$ This was a genetic substudy of the Trial to Assess Improvement in Therapeutic Outcomes by Optimizing Platelet Inhibition with PrasugrelThrombolysis in Myocardial Infarction (TRITONTIMI 38) trial in which patients with ACS with planned PCI were randomly assigned to receive a clopidogrel 300-mg loading dose followed by 75 $\mathrm{mg}$ /day for up to 15 months or prasugrel (a new thienopyridine). In the 1477 patients assigned to clopidogrel and who provided a genetic sample, genotyping was performed for polymorphisms in the genes encoding CYP2C19, CYP2C9, CYP2B6, CYP3A5, CYP3A4, and CYP1A2 enzymes. The rate of adverse cardiovascular outcomes, which consisted of death from cardiovascular causes, myocardial infarction, or stroke, was compared among genotype groups. Risk of stent thrombosis and safety outcomes (TIMI major and minor bleeding not related to coronary artery bypass grafting) were also assessed. Those possessing at least one CYP2C19 reduced- 
function allele (395 patients) had an increased risk for the primary outcome compared with wild-type homozygotes. Patients possessing at least one reduced-function CYP2C19 allele also had 3 times the risk for stent thrombosis compared with noncarriers. Genetic variation in the other CYP enzymes studied was not associated with the primary outcome, and no association was seen between bleeding risk and CYP genotype. This study was not designed to assess whether possession of two reducedfunction CYP2C19 alleles is associated with risk of the primary outcome; therefore, we do not know whether carriers and homozgygotes had different event rates. This study further confirms that patients carrying at least one variant CYP2C19 allele have an increased risk for adverse cardiovascular events after PCI.

However, reports from substudies of the Impact of the Extent of Clopidogrel-Induced Platelet Inhibition During Elective Stent Implantation on Clinical Event Rate (EXCELSIOR) and Clopidogrel as Adjunctive Reperfusion Therapy-Thrombolysis in Myocardial Infarction (CLARITY-TIMI 28) trials did not find an association between CYP2C19 genotype and clopidogrel responsiveness. ${ }^{16,17}$ A genetic substudy of EXCELSIOR was the first large cohort study to evaluate the role of CYP2C19 genotype in clopidogrel responsiveness. ${ }^{16}$ The primary aim was to assess whether the loss-offunction CYP2C19*2 polymorphism was associated with elevated platelet reactivity in patients receiving clopidogrel therapy. The association between this measure of elevated platelet reactivity and 1-year occurrence of death or myocardial infarction was also assessed. Seven hundred ninety-seven patients underwent elective coronary stent placement and received a 600-mg loading dose of clopidogrel and aspirin therapy. Platelet aggregation was assessed by ex vivo optical aggregation induced by adenosine $5^{\prime}$-diphosphate immediately after the loading dose of clopidogrel and before discharge 2-4 hours after the first clopidogrel maintenance dose. Patients carrying a variant CYP2C19*2 allele were more likely to have elevated platelet aggregation, after both the loading and maintenance doses of clopidogrel, compared with wild-type homozygotes (before discharge $41.3 \%$ vs $22.5 \%, p<0.001$ ), but the study did not find an association between CYP2C19 genotype and death or myocardial infarction. However, patients with elevated platelet aggregation before discharge had a 3-fold increase in the 1-year occurrence of death or myocardial infarction.

A genetic substudy of CLARITY-TIMI 28 had similar results. ${ }^{17}$ In CLARITY-TIMI 28, a total of 3941 patients received fibrinolytic therapy for STsegment elevation myocardial infarction and were randomly assigned to receive a clopidogrel 300mg loading dose followed by $75 \mathrm{mg}$ once/day or placebo. Patients were then scheduled to undergo angiography 48-192 hours after study entry and were followed for 30 days. Four hundred sixty-five patients participated in a substudy in which genotyping was performed for polymorphisms in the genes encoding CYP1A2, CYP2C9, CYP2C19, CYP3A4, and CYP3A5 enzymes. The primary end point assessed was a composite of an occluded infarct-related artery at angiography, death before angiography could be performed, or recurrent myocardial infarction before angiography. None of the genotypes studied were associated with the primary composite end point.

\section{CYP3A4 and CYP3A5}

The CYP3A4 and CYP3A5 enzymes also play a role in the conversion of clopidogrel to its active metabolite. The CYP3A family is the most prolifically expressed CYP enzyme family in human hepatocytes. However, CYP3A4 and CYP3A5 levels differ by race, with Caucasians expressing primarily CYP3A4 and AfricanAmericans expressing CYP3A5. There is a significant amount of overlap in CYP3A4 and CYP3A5 substrates, and they are responsible for the metabolism of approximately half of the drugs used today. Although polymorphisms in CYP3A4 have been extensively studied, no relatively common variants have been linked conclusively to a change in gene expression or drug metabolism (Table 1). ${ }^{22}$ There are, however, variants of functional consequence in CYP3A5. The CYP3A5*3 variant allele has been associated with decreased functional CYP3A activity, and patients who possess two CYP3A5*3 alleles have approximately 50\% less CYP3A activity compared with $C Y P 3 A 5^{*} 1$ (wild-type) homozygotes. The allele frequency for CYP3A5*3 is $85 \%$ among Caucasians, 55\% among African-Americans, and $65 \%$ among Chinese. ${ }^{22,23}$

Because of the number of variants in both CYP3A4 and CYP3A5 and their role in clopidogrel activation, polymorphisms in both genes have also been studied for their role in clopidogrel responsiveness. Several studies have 
assessed changes in clopidogrel pharmacokinetics and pharmacodynamics associated with CYP3A4 genotype. However, only one study found a positive association between CYP3A4 genotype and the effect of clopidogrel on platelet aggregation, not clinical outcomes. ${ }^{24}$ This study, however, assessed only the role of the CYP3A4 genotype in clopidogrel responsiveness; polymorphisms in CYP2C19 were not assessed. Most studies have found no association between CYP3A4 genotype and clopidogrel responsiveness. ${ }^{14,}$ 17, 25-27 In fact, when CYP3A4 genotype was assessed in the large, outcomes-based study of TRITONTIMI 38, in conjunction with CYP2C19, 2C9, $2 B 6,3 A 5$, and $1 A 2$, no association was found. ${ }^{15}$

The results for the better characterized CYP3A5 polymorphisms have been slightly different. One group of investigators found that patients who possess the CYP $3 A 5 * 3$ (nonexpressor) allele had similar platelet aggregation with clopidogrel compared with those possessing the CYP $3 A 5^{*} 1{ }^{*} 1$ genotype. ${ }^{28}$ However, subjects with the CYP3A5*3 allele had significantly decreased response to clopidogrel when it was combined with itraconazole, a CYP3A inhibitor, compared with CYP3A $5^{*} 1$ homozygotes. In addition, they found that possession of the CYP $3 A 5 * 3$ allele and increased number of coadministered CYP3A substrates and inhibitors were associated with an increased risk of atherothrombotic events. However, other studies have demonstrated that the association between CYP3A genotype and clopidogrel response does not appear to be as strong as that found with CYP2C19 genotype. ${ }^{14,25 \text {, }}$ 26, 29 In fact, most studies assessing CYP3A4 or CYP3A5 and CYP2C19 genotypes have found an association only with CYP2C19 genotype and clopidogrel responsiveness.

\section{$A B C B 1$}

P-glycoprotein (P-gp) is an efflux transport protein located in many tissues in the body. ${ }^{30}$ These tissues include the surface of the small and large intestine, the renal proximal tubules, and the blood-brain barrier. In the small and large intestines, P-gp pumps drugs back into the intestinal lumen, thus limiting bioavailability. The P-gp is just one of several ABCB superfamily transporters. Genetic variation in the gene encoding P-gp $(A B C B 1)$ has been well studied; however, the functional consequence of polymorphisms in ABCB1 is unclear (Table 1). Some studies have found an association between protein expression and drug transport with
$A B C B 1$ polymorphisms, but these associations have not been demonstrated consistently.

The P-gp is involved in clopidogrel absorption, and the role of $A B C B 1$ polymorphisms in clopidogrel responsiveness has been well studied. Three ABCB1 single nucleotide polymorphisms (C3435T, G2677T, and C136T) have been evaluated for their role in clopidogrel pharmacokinetics. ${ }^{31}$ Lower clopidogrel concentrations (both maximum concentration and area under the concentration-time curve) after a single dose of 300 or $600 \mathrm{mg}$ were noted in patients who were homozygous for the variant $A B C B 13435 \mathrm{~T}$ allele. Of interest, a larger loading dose of 900 mg overcame this difference. In addition, results from the FAST-MI study suggest that subjects with two variant $A B C B 13435 \mathrm{~T}$ alleles are at increased risk for clinically documented clopidogrel resistance, in the form of death, nonfatal stroke, or myocardial infarction (Table 2). ${ }^{19}$ Of note, in this study, consideration of $A B C B 1$ genotype combined with CYP2C19 genotype improved prediction of clopidogrel nonresponsiveness. Unfortunately, none of the other large cohort studies assessed the role of $A B C B 1$ genotyping in addition to CYP2C19.

\section{P2RY12}

Studies have also assessed genetic variation in the gene encoding the $\mathrm{P}_{2} \mathrm{Y}_{12}$ receptor (the active site for clopidogrel), P2RY12. Polymorphisms in P2RY12 have not been consistently associated with clopidogrel response as measured by either platelet aggregation or clinical events (Table 1). ${ }^{32-34}$ The P2RY12 genotype was also assessed in the FAST-MI study, and no association was found with clopidogrel responsiveness. ${ }^{19}$

The pharmacogenetic literature further supports the importance of the role of CYP2C19 in the metabolism of clopidogrel, initially suggested by the drug interaction literature. ${ }^{35}$ These data would also suggest that polymorphisms in $A B C B 1$ may be associated with a decrease in clopidogrel responsiveness.

\section{Clinical Relevance}

Patients at Risk for Decreased Response to Clopidogrel

Approximately 2-3\% of Caucasian and AfricanAmerican patients and $10-15 \%$ of Asian patients possessing two variant CYP2C19 alleles will have impaired clopidogrel responsiveness. The data would also suggest that patients possessing only 
one CYP2C19 loss-of-function allele may be at increased risk. Approximately $25 \%$ of Caucasians, $30 \%$ of African-Americans, and $50 \%$ of Asians possess at least one variant allele. Also, patients possessing two variant $A B C B 13435 \mathrm{~T}$ alleles may be more likely to have decreased clopidogrel responsiveness. In fact, patients possessing both a variant CYP2C19 allele and two variant $A B C B 1$ $3435 \mathrm{~T}$ alleles may be at the highest risk for clopidogrel nonresponsiveness.

Routine Genotyping for CYP2C19 Variant Alleles to Predict Clopidogrel Responsiveness: Logistic and Cost Considerations

The updated clopidogrel label now includes information on the role of CYP2C19 genotype in clopidogrel responsiveness. ${ }^{10}$ Specifically, the label states "...CYP2C19 poor metabolizer status is associated with diminished response to clopidogrel. The optimal dose regimen for poor metabolizers has yet to be determined." 10 Although CYP2C19 genotyping is currently available, this test often requires the use of a specialty laboratory and generally is sent out with a turnaround time of 6-10 days. Genotyping for CYP2C19 is also quite expensive, with a list price of \$395-500 and is not being covered by insurance companies. Currently, $A B C B 1$ genotyping is not available. There are also logistic issues associated with genotyping an ACS population. Clopidogrel therapy is often started immediately upon presentation of the patient to the emergency department, so there is little time for genetic testing before initiation of therapy. If genotyping were to be done in this setting, a rapid and clinically useful platform would be necessary.

In addition, all of the variability in clopidogrel responsiveness is clearly not captured by genotyping only for CYP2C19 loss-of-function alleles. This is partly because genetic variability in $A B C B 1$ and CYP3A4 are not being assessed. Few CYP3A4 polymorphisms have been shown to have functional effects. Therefore, there is no good way through genotyping to characterize the variability in CYP3A4 metabolic activity. In addition, whereas the $A B C B 1$ genotype may contribute to prediction of clopidogrel nonresponsiveness, $A B C B 1$ genotyping is not available. It is also likely that drug interactions through the CYP system (such as those discussed in the drug interaction literature ${ }^{35}$ ) serve as an additional source of variability in clopidogrel responsiveness. The amount of interindividual variability in clopidogrel responsiveness remaining after CYP2C19 genotyping may limit the utility of genotyping.

An alternative $\mathrm{P} 2 \mathrm{Y}_{12}$ receptor inhibitor is available, and others may be available soon. Prasugrel was recently approved by the United States Food and Drug Administration for use in combination with aspirin in patients with ACS who will be managed with PCI. Prasugrel is also a prodrug, and its active metabolite has similar affinity for the $\mathrm{P}_{2} \mathrm{Y}_{12}$ receptor as does the active metabolite of clopidogrel. ${ }^{36}$ However, whereas clopidogrel undergoes two activation steps through the CYP system, prasugrel requires only one. This drug appears to have little propensity for drug interactions or susceptibility to CYP genetic variation, so it may be an attractive treatment option in patients for whom clopidogrel therapy is indicated. This may be even more true when clopidogrel nonresponsiveness is predicted or measured by platelet aggregation testing. ${ }^{36}$ There are several point-of-care tests available that measure ex vivo platelet aggregation, and they may be used clinically. The utility of these tests has been reviewed previously. $7,8,37,38$

Compared with clopidogrel, prasugrel is associated with an increased risk of bleeding. ${ }^{39}$ In addition, prasugrel is contraindicated in patients with a previous transient ischemic attack or stroke, but careful consideration should be given to patients aged 75 years or older and weighing less than $60 \mathrm{~kg}$. The retrospective data from the TRITON-TIMI 38 study suggest that patients aged 75 years or older with a history of diabetes mellitus or myocardial infarction can be treated with prasugrel $10 \mathrm{mg} /$ day, and a daily dose of $5 \mathrm{mg}$ for those weighing less than $60 \mathrm{~kg}$ can be beneficial, but this has not been tested prospectively. ${ }^{39}$ However, the true excess bleeding risk with prasugrel is difficult to determine because approximately $30 \%$ of patients receiving clopidogrel are nonresponsive and therefore have no bleeding risk, creating an unequal reference population. In addition, we found no study that evaluated whether switching patients to prasugrel when they have been classified as clopidogrel nonresponsive leads to improved outcomes. It is therefore unclear how much advantage prasugrel therapy provides, which is particularly important given the increased risk of bleeding.

In the future, CYP2C19 genotype or platelet aggregation test results could be used to predict a subset of patients most likely to respond to clopidogrel. This may ultimately become the 
most cost-effective strategy for society, given that clopidogrel will become generic in 2012.

\section{Conclusion}

There is a considerable amount of interindividual variability in clopidogrel responsiveness. The literature suggests that CYP2C19 polymorphisms and, to a lesser extent, $A B C B 1$ polymorphisms contribute to this variability. Specifically, patients possessing at least one variant CYP2C19 allele (CYP2C19*2, *3) have impaired clopidogrel responsiveness due to decreased formation of the active metabolite. In addition, one study found that considering the $A B C B 1$ genotype in addition to CYP2C19 allowed better prediction of clopidogrel nonresponsiveness. However, routine genotyping for CYP2C19 or ABCB1 polymorphisms in order to predict clopidogrel response cannot be recommended because of logistic and cost considerations.

\section{References}

1. CAPRIE Steering Committee. A randomised, blinded trial of clopidogrel versus aspirin in patients at risk of ischaemic events (CAPRIE). Lancet 1996;348:1329-39.

2. Yusuf S, Zhao F, Mehta SR, et al. Effects of clopidogrel in addition to aspirin in patients with acute coronary syndromes without ST-segment elevation. N Engl J Med 2001;345: 494-502.

3. Steinhubl SR, Berger PB, Mann JT III, et al. Early and sustained dual oral antiplatelet therapy following percutaneous coronary intervention: a randomized controlled trial. JAMA 2002;288:2411-20.

4. Anderson JL, Adams CD, Antman EM, et al. ACC/AHA 2007 guidelines for the management of patients with unstable angina/non-ST-elevation myocardial infarction: a report of the American College of Cardiology/American Heart Association task force on practice guidelines (writing committee to revise the 2002 guidelines for the management of patients with unstable angina/non-ST-elevation myocardial infarction) developed in collaboration with the American College of Emergency Physicians, the Society for Cardiovascular Angiography and Interventions, and the Society of Thoracic Surgeons endorsed by the American Association of Cardiovascular and Pulmonary Rehabilitation and the Society for Academic Emergency Medicine. J Am Coll Cardiol 2007;50:el-157.

5. King SB III, Smith SC Jr, Hirshfeld JW Jr, et al. 2007 focused update of the ACC/AHA/SCAI 2005 guideline update for percutaneous coronary intervention: a report of the American College of Cardiology/American Heart Association task force on practice guidelines. J Am Coll Cardiol 2008;51:172-209.

6. Reaume KT, Regal RE, Dorsch MP. Indications for dual antiplatelet therapy with aspirin and clopidogrel: evidencebased recommendations for use. Ann Pharmacother 2008;42: $550-7$.

7. Gurbel PA, Becker RC, Mann KG, Steinhubl SR, Michelson AD. Platelet function monitoring in patients with coronary artery disease. J Am Coll Cardiol 2007;50:1822-34.

8. Price MJ. Bedside evaluation of thienopyridine antiplatelet therapy. Circulation 2009;119:2625-32.

9. Angiolillo DJ, Fernandez-Ortiz A, Bernardo E, et al. Variability in platelet aggregation following sustained aspirin and clopidogrel treatment in patients with coronary heart disease and influence of the $807 \mathrm{C} / \mathrm{T}$ polymorphism of the glycoprotein Ia gene. Am J Cardiol 2005;96:1095-9.

10. sanofi-aventis and Bristol-Myers Squibb. Plavix (clopidogrel) package insert. Bridgewater, NJ; 2009.

11. Desta Z, Zhao X, Shin JG, Flockhart DA. Clinical significance of the cytochrome P450 2C19 genetic polymorphism. Clin Pharmacokinet 2002;41:913-58.

12. Xie HG, Kim RB, Wood AJ, Stein CM. Molecular basis of ethnic differences in drug disposition and response. Annu Rev Pharmacol Toxicol 2001:41:815-50.

13. Kim KA, Park PW, Hong SJ, Park JY. The effect of CYP2C19 polymorphism on the pharmacokinetics and pharmacodynamics of clopidogrel: a possible mechanism for clopidogrel resistance. Clin Pharmacol Ther 2008;84:236-42.

14. Giusti B, Gori AM, Marcucci R, et al. Cytochrome P450 2C19 loss-of-function polymorphism, but not CYP3A4 IVS10 + 12G/A and P2Y12 T744C polymorphisms, is associated with response variability to dual antiplatelet treatment in high-risk vascular patients. Pharmacogenet Genomics 2007;17:1057-64.

15. Mega JL, Close SL, Wiviott SD, et al. Cytochrome P450 polymorphisms and response to clopidogrel. N Engl J Med 2009;360:354-62.

16. Trenk D, Hochholzer W, Fromm MF, et al. Cytochrome P450 2C19 681G>A polymorphism and high on-clopidogrel platelet reactivity associated with adverse l-year clinical outcome of elective percutaneous coronary intervention with drug-eluting or bare-metal stents. J Am Coll Cardiol 2008;51:1925-34.

17. Mega JL, Thakuria JV, Cannon CP, Sabatine MS. Sequence variations in CYP metabolism genes and cardiovascular outcomes following treatment with clopidogrel: insights from the CLARITY-TIMI 28 genomic study [abstract]. J Am Coll Cardiol 2008;51:A206

18. Collet JP, Hulot JS, Pena A, et al. Cytochrome P450 2C19 polymorphism in young patients treated with clopidogrel after myocardial infarction: a cohort study. Lancet 2009;373:309-17.

19. Simon T, Verstuyft C, Mary-Krause M, et al. Genetic determinants of response to clopidogrel and cardiovascular events. N Engl J Med 2009;360:363-75.

20. Sibbing D, Stegherr J, Latz W, et al. Cytochrome P450 2C19 loss-of-function polymorphism and stent thrombosis following percutaneous coronary intervention. Eur Heart J 2009;30: 916-22.

21. Giusti B, Gori AM, Marcucci R, et al. Relation of cytochrome P450 2C19 loss-of-function polymorphism to occurrence of drug-eluting coronary stent thrombosis. Am J Cardiol 2009;103:806-11.

22. Ingelman-Sundberg M, Sim SC, Gomez A, Rodriguez-Antona C. Influence of cytochrome $\mathrm{P} 450$ polymorphisms on drug therapies: pharmacogenetic, pharmacoepigenetic and clinical aspects. Pharmacol Ther 2007;116:496-526.

23. Kuehl P, Zhang J, Lin Y, et al. Sequence diversity in CYP3A promoters and characterization of the genetic basis of polymorphic CYP3A5 expression. Nat Genet 2001;27:383-91.

24. Angiolillo DJ, Fernandez-Ortiz A, Bernardo E, et al. Contribution of gene sequence variations of the hepatic cytochrome P450 3A4 enzyme to variability in individual responsiveness to clopidogrel. Arterioscler Thromb Vasc Biol 2006;26:1895-900.

25. Frere C, Cuisset T, Morange PE, et al. Effect of cytochrome P450 polymorphisms on platelet reactivity after treatment with clopidogrel in acute coronary syndrome. Am J Cardiol 2008;101:1088-93.

26. Geisler T, Schaeffeler E, Dippon J, et al. CYP2C19 and nongenetic factors predict poor responsiveness to clopidogrel loading dose after coronary stent implantation. Pharmacogenomics 2008;9:1251-9.

27. Varenhorst C, James S, Erlinge D, et al. Genetic variation of CYP2C19 affects both pharmacokinetic and pharmacodynamic responses to clopidogrel but not prasugrel in aspirin-treated patients with coronary artery disease. Eur Heart J 2009;30: 1744-52.

28. Suh JW, Koo BK, Zhang SY, et al. Increased risk of atherothrombotic events associated with cytochrome P450 3A5 
polymorphism in patients taking clopidogrel. CMAJ 2006;174:1715-22.

29. Hulot JS, Bura A, Villard E, et al. Cytochrome P450 2C19 lossof-function polymorphism is a major determinant of clopidogrel responsiveness in healthy subjects. Blood 2006;108:2244-7.

30. DuBuske LM. The role of P-glycoprotein and organic aniontransporting polypeptides in drug interactions. Drug Saf 2005;28:789-801.

31. Taubert D, vonBeckerath N, Grimberg G, et al. Impact of Pglycoprotein on clopidogrel absorption. Clin Pharmacol Ther 2006;80:486-501.

32. Ziegler S, Schillinger M, Funk M, et al. Association of a functional polymorphism in the clopidogrel target receptor gene, P2Y12, and the risk for ischemic cerebrovascular events in patients with peripheral artery disease. Stroke 2005;36:1394-9.

33. Cuisset T, Frere C, Quilici J, et al. Role of the T744C polymorphism of the P2Y12 gene on platelet response to a 600mg loading dose of clopidogrel in 597 patients with non-STsegment elevation acute coronary syndrome. Thromb Res
2007;120:893-9.

34. Angiolillo DJ, Fernandez-Ortiz A, Bernardo E, et al. Lack of association between the $\mathrm{P}_{2} \mathrm{Y}_{12}$ receptor gene polymorphism and platelet response to clopidogrel in patients with coronary artery disease. Thromb Res 2005;116:491-7.

35. Liu TJ, Jackevicius CA. Drug interaction between clopidogrel and proton pump inhibitors 2010;30:275-89.

36. Mega JL, Close SL, Wiviott SD, et al. Cytochrome P450 genetic polymorphisms and the response to prasugrel: relationship to pharmacokinetic, pharmacodynamic, and clinical outcomes. Circulation 2009;119:2553-60.

37. Oestreich JH, Smyth SS, Campbell CL. Platelet function analysis: at the edge of meaning. Thromb Haemost 2009;101: 217-19.

38. Mahla E, Antonino MJ, Tantry US, Gurbel PA. Point-of-care platelet function analysis ready for prime time? J Am Coll Cardiol 2009;53:857-9.

39. Wiviott SD, Braunwald E, McCabe $\mathrm{CH}$, et al. Prasugrel versus clopidogrel in patients with acute coronary syndromes. N Engl J Med 2007;357:2001-15. 